\title{
Advantages of Japanese response criteria for estimating the survival of patients with primary gastric cancer
}

\author{
Wasaburo Koizumi ${ }^{1}$, Minoru Kurihara², Satoshi Tanabe ${ }^{1}$, Ichiei Kondo ${ }^{1}$, Izumi Yamazaki ${ }^{1}$, Miwa Nonaka ${ }^{3}$, \\ Yoshiki Shimamura ${ }^{4}$, Katsunori Saigenji ${ }^{1}$ and the Advanced Gastric Cancer Cooperative Study Group \\ ${ }^{1}$ Department of Gastroenterology, Kitasato University East Hospital, School of Medicine, 2-1-1 Asamizodai, Sagamihara, \\ Kanagawa 228-8520, Japan \\ ${ }^{2}$ Department of Gastroenterology, Toyosu Hospital, Showa University, 4-1-18 Toyosu, Kotoku, Tokyo 135-8577, Japan \\ ${ }^{3}$ Department of Pharmacy, Kitasato University East Hospital, 2-1-1 Asamizodai, Sagamihara, Kanagawa 228-8520, Japan \\ ${ }^{4}$ Department of Clinical Biochemistry, School of Pharmacy, Tokyo University of Pharmacy and Life Science, 1432-1 Horinouchi, Hachioji, \\ Tokyo 192-0392, Japan
}

\begin{abstract}
:
Background. We conducted a retrospective study to investigate the adequacy of the Efficacy Criteria for Primary Lesions in the Japanese Classification of Gastric Cancer (Japanese criteria) for evaluating the anti-tumor efficacy of chemotherapies and the relationship between tumor regression and the prognosis of gastric cancer.

Methods. The data for 90 patients with inoperable advanced gastric cancer who received various chemotherapies, consisting of fluorinated pyrimidines and cisplatin, were retrospectively analyzed. Based on the Japanese criteria, we investigated the efficacy of the chemotherapies and the relationship between the response in primary lesions and survival. We also compared the efficacy of chemotherapies evaluated by the Japanese criteria to that evaluated by the WHO criteria.

Results. All 90 patients were evaluable by the Japanese criteria. The overall response rate was $53.3 \%$ (Partial response [PR] in 48 patients and no change + progressive disease [NC $+\mathrm{PD}]$ in 42 patients). The primary lesions were classified as measurable (a-lesions) in 27 patients, evaluable but not measurable (b-lesions) in 31 patients, and diffusely infiltrating (clesions) in 32 patients. Overall median survival time (MST) was 9.4 months. The MSTs of the responders and nonresponders were $\mathbf{1 2 . 6}$ and $\mathbf{7 . 8}$ months, respectively. In contrast, by the WHO criteria, 49 patients $(54.4 \%)$ were evaluable; the other 41 patients had gastric primary lesions alone but were not measurable by WHO criteria. The overall response rate was $67.3 \%(33 / 49)$, and overall MST was 9.4 months. The MSTs of the responders evaluated by both sets of criteria were both 12.6 months.

Conclusions. We suggest that the Japanese criteria are useful for evaluating the anti-tumor effect of gastric cancer chemotherapies and that prospective studies to reconfirm their usefulness are warranted in Japan, and in Western countries.
\end{abstract}

Offprint requests to: $\mathrm{W}$. Koizumi

Received for publication on April 20, 1998; accepted on Jan. 19, 1999
Key words: inoperable, gastric cancer, chemotherapy, efficacy criteria, primary lesions

\section{Introduction}

Assessments of the efficacy of various therapies for malignant tumors are generally done in accordance with the criteria of the National Cancer Institute (NCI) [1], the Eastern Cooperative Oncology Group (ECOG) [2], the International Union against Cancer (UICC) [3], or the World Health Organization (WHO) [4]. In Japan, common criteria for dealing with malignant tumors were created, based on these criteria. Individual criteria for various types of tumor were prepared based on the common criteria. Criteria for the assessment of response to treatment for gastric cancer are described in the Japanese classification of gastric cancer (1985) [5], which was partly revised in 1993 with an English edition in 1995 [6,7]. Among these criteria, tumor regression has been determined to be an index for anti-tumor efficacy, but the relationship between tumor regression and survival prognosis has been debated. Both prolongation of survival and improvement in quality of life are important endpoints in cancer therapy. Assessment of the anti-tumor efficacy of a therapy is important in determining whether the therapy should be continued. It is ideal that the assessment could also be capable of predicting patient survival. In gastric cancer, measurement of tumor regression is difficult, not only because the organ is characteristically luminal but because lesions are frequently diffusely infiltrating. Internationally, only metastatic lesions, not primary lesions, are assessed for most patients. In Japan, therapies for gastric cancer have been assessed in accordance with the Efficacy Criteria for Primary Lesions in the Japanese Classification of Gastric Cancer - Response Assessment of Chemotherapy for Gastric Cancer (Japanese criteria) 
Table 1. Patient characteristics

\begin{tabular}{lc}
\hline Characteristics & No. of patients \\
\hline Median age (range) (years) & $58(31-85)$ \\
Sex & 34 \\
Female & 56 \\
Male & \\
Performance status & 2 \\
0 & 34 \\
1 & 37 \\
2 & 17 \\
3 & \\
Extent of disease & 22 \\
Locally advanced & 68 \\
With distant metastasis & \\
Primary lesion & 27 \\
a-lesion & 31 \\
b-lesion & 32 \\
c-lesion & \\
Chemotherapy regimens & 28 \\
Fluorinated pyrimidines + cisplatin & \\
Fluorinated pyrimidines + cisplatin + & 44 \\
$\quad$ mitomycin C & \\
Other regimens mainly consisting of & \\
$\quad$ fluorinated pyrimidines & \\
\hline
\end{tabular}

[5-7]. The purpose of this retrospective study was to investigate the appropriateness of these criteria for evaluating the anti-tumor efficacy of gastric cancer therapies, through a comparison of the efficacy of chemotherapies evaluated by the Japanese criteria and the WHO criteria.

\section{Patients and methods}

Ninety patients with advanced gastric cancer, who were diagnosed with highly infiltrated tumors or distant metastases which precluded operation, were retrospectively analyzed. Patient characteristics are shown in Table 1.

The chemotherapy regimens consisted of fluorinated pyrimidines + cisplatin $(n=28)$, fluorinated pyrimidines + cisplatin + mitomycin $\mathrm{C}(n=44)$, with other regimens mainly consisting of fluorinated pyrimidines $(n=18)$. All these patients underwent upper gastrointestinal X-ray examination, endoscopy, and computed tomography (CT) scans prior to treatment. Based on the results of these examinations, lesions were classified as measurable (a-lesions), evaluable but not measurable (b-lesions) and diffusely infiltrating (clesions) in accordance with the Japanese criteria. An outline of the efficacy assessment is shown in Table 2 and Fig. 1. X-ray and endoscopic examinations of the upper gastrointestinal tract were performed every 4
Table 2. Japanese response assessment criteria for primary lesions of gastric cancer ${ }^{\mathrm{a}}$

Measurable lesions (a-lesions)

Evaluation scale: total tumor size on X-ray and/or endoscopic examination

CR: Complete disappearance, continuously for more than 4 weeks

PR: More than 50\% decrease, continuously for more than 4 weeks

NC: Less than $50 \%$ decrease or less than $25 \%$ increase for more than 4 weeks

PD: More than 25\% increase or the appearance of new lesions

Evaluable but not measurable lesions (b-lesions)

Evaluation scale: macroscopic change on X-ray and/or endoscopic examination

CR: Complete disappearance, continuously for more than 4 weeks

PR: Clearly differ from pretreatment examination, for more than 4 weeks, e.g., showing marked regression and flattening of elevated or ulcerated lesions (estimated decrease of more than 50\%)

NC: No change in comparison with pretreatment findings or no change appropriate for categorization as PR, for more than 4 weeks

PD: Progression or the appearance of new lesions

Diffusely infiltrating lesions (c-lesions)

Evaluation scale: Expansion of affected gastric lumen on $\mathrm{X}$-ray examination and/or endoscopic examinations

CR: All lesions have disappeared

PR: Gastric lumen of the affected region on the X-ray shows enlargement of more than $50 \%$, continuously for more than 4 weeks

NC: Unchanged or enlarged by less than $50 \%$, for more than 4 weeks

PD: Evidence of worsening disease or new lesions

${ }^{a}$ Excerpted from reference 7

Also see Fig. 1

$\mathrm{CR}$, Complete response; $\mathrm{PR}$, partial response; NC, no change; PD, progressive disease

weeks for efficacy assessment. All efficacy and safety assessments were performed by at least three extramural oncologists. Informed consent to receive treatment and undergo the various examinations was obtained from all patients. The prognosis for all patients was investigated.

Statistical analyses were performed with Statistical Analysis System (SAS, Cary, NC, USA) software. Survival curves were generated by the Kaplan-Meier method. The log-rank test was used to compare survival distributions. Hazard ratios were estimated using Cox's proportional hazard model [8].

\section{Results}

All 90 patients were evaluable for efficacy evaluation by the Japanese criteria. 
a-lesions
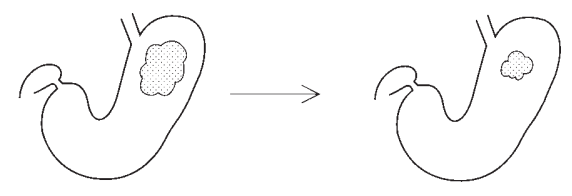

b-lesions
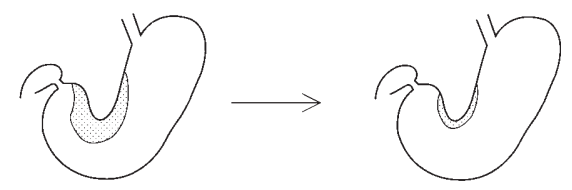

c-lesions
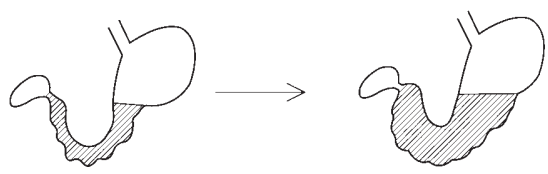

Fig. 1. Pictures illustrating Japanese response criteria for primary lesions of gastric cancer. Measurable lesions ( $a$ lesions): More than $50 \%$ decrease of total tumor size on X-ray and/or endoscopic examination, for more than 4 weeks. Evaluable but not measurable lesions (b-lesions): Findings clearly differ from pretreatment examination, e.g., showing marked regression and flattening of elevated or ulcerated lesions (estimated decrease of more than 50\%) on macroscopic change on X-ray and/or endoscopic examination, for more than 4 weeks. Diffusely infiltrating lesions $(c$ lesions): Enlargement of more than $50 \%$ of the gastric lumen of the affected region (gray-shaded area) on X-ray examination and/or endoscopic examination, for more than 4 weeks

The primary lesions were classified as follows: alesions: 27 patients, b-lesions: 31 patients, and c-lesions: 32 patients. The overall response rate to the treatments in these 90 patients was $53.3 \%$; there was a partial response in 48 patients (responders) and no change + progressive disease in 42 patients (non-responders). The response rate for a-lesions was $85.2 \%$ (23/27 patients), that for b-lesions, 38.7\% (12/31 patients); and that for c-lesions, $40.6 \%$ (13/32 patients). The highest response rate was observed for a-lesions.

The overall median survival time (MST) was 9.4 months. The MSTs classified by lesions were as follows: a-lesions, 13.6 months; b-lesions, 8.6 months; and clesions, 8.4 months. MST was longest in patients with a-lesions.

Relationships between the response and survival time were investigated. The MST of the non-responders was 7.8 months, while responders showed a significantly superior MST, 12.6 months $(P=0.0001)$.

Figures 2 and 3 show the Kaplan-Meier curves for responders and non-responders, respectively, according to type of lesion. The MSTs of responders with $\mathrm{a}-, \mathrm{b}-$, and c-lesions were 14.2, 11.5, and 10.5 months, respectively.

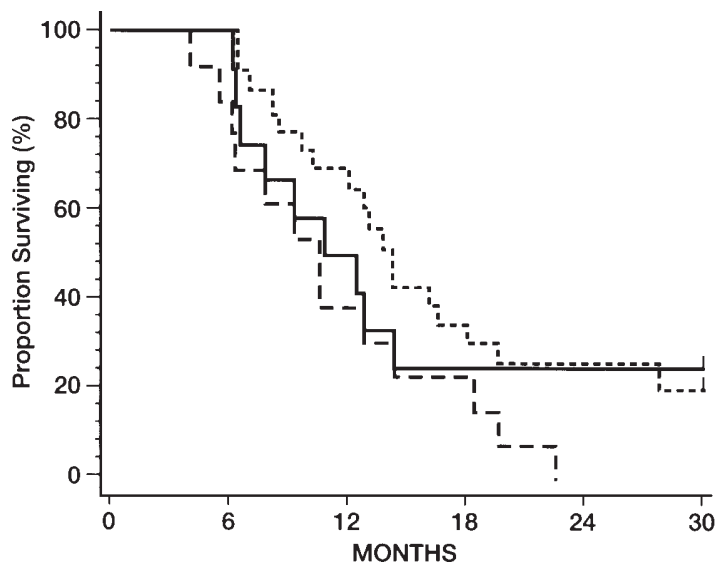

Fig. 2. Kaplan-Meier curves comparing median survival time (MST; months) and 1-year survival (\%) in responders with a(dotted line; $n=23$; MST, 14.2; 1-year survival, 65.2\%) b(continuous line; $n=12$; MST, 11.5; 1 -year survival, $50 \%$ ) and c-lesions (dashed line; $n=13$; MST, 10.5; 38.5\%)

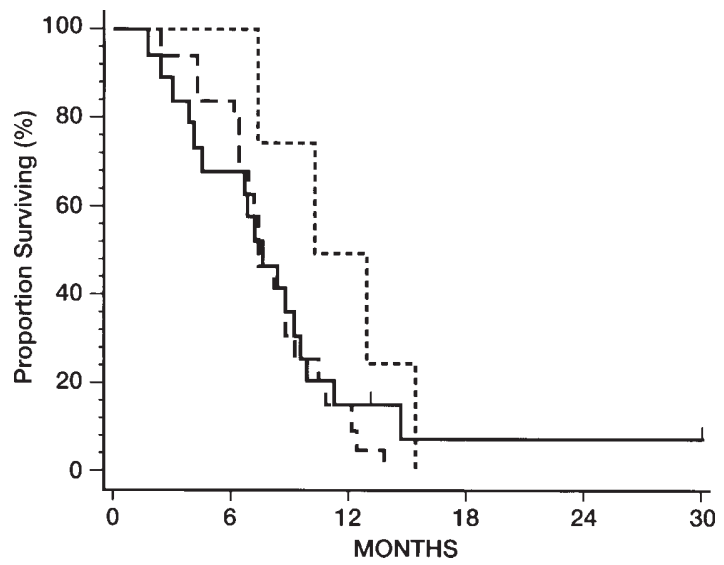

Fig. 3. Kaplan-Meier estimates of the probability of survival comparing non-responders with a- (dotted line; $n=4$; MST, 11.4; 1 -year survival, $50 \%$ ) b- (continuous line; $n=19$; MST, 7.4; 1-year survival, 15.8\%) and c-lesions (dashed line; $n=19$; MST, 7.2; 1-year survival, 10.5\%). Lines As in Fig. 2

To quantify the difference in MSTs, hazard ratios were estimated using Cox's proportional hazard model. The hazard ratios with b- or c-lesions, respectively, were 1.79 and 2.19 (hazard ratio with a-lesions $=1.00$ ). The MSTs of non-responders with a-, b-, or c-lesions were $11.4,7.4$, and 7.2 months, respectively, with hazard ratios being 1.29 with b-lesions and 2.00 with c-lesions (hazard ratio with a-lesions $=1.00$ ).

When the WHO criteria were employed for efficacy evaluation, the number of evaluable patients decreased to $49(54.4 \%)$, because the remaining 41 patients had gastric primary lesions alone, which were not measur- 
Table 3. Comparison of the number of evaluable and/or measurable patients by the Japanese criteria and WHO criteria

\begin{tabular}{lll}
\hline & $\begin{array}{c}\text { Japanese } \\
\text { criteria }^{7}\end{array}$ & $\begin{array}{c}\text { WHO } \\
\text { criteria }^{4}\end{array}$ \\
\hline $\begin{array}{l}\text { Total number of patients } \\
\text { No. of evaluable and / or } \\
\text { measurable patients }\end{array}$ & 90 & 90 \\
$\begin{array}{l}\text { Responders } \\
\text { Non-responders }\end{array}$ & $40(53.3 \%)$ & 49 \\
\hline
\end{tabular}

Table 4. Median survival time (MST) of evaluable and/or measurable patients by Japanese criteria and WHO criteria

\begin{tabular}{lccrrr}
\hline & \multicolumn{2}{c}{ Japanese criteria $^{7}$} & & \multicolumn{2}{c}{ WHO criteria } \\
\cline { 2 - 3 } \cline { 5 - 6 } & $n$ & MST (months) & & $n$ & MST (months) \\
\hline Overall & 90 & 9.4 & & 49 & 9.4 \\
Responders & 48 & 12.6 & & 33 & 12.6 \\
Non-responders & 42 & 7.8 & & 16 & 7.6 \\
\hline
\end{tabular}

able by the WHO criteria; the overall response rate was $67.3 \%$ (33/49) (Table 3) and overall MST was 9.4 months (Table 4). Figure 4 shows the Kaplan-Meier curves of responders evaluated by the Japanese criteria and the WHO criteria. Interestingly, the MSTs of responders evaluated by the Japanese criteria and the WHO criteria were both 12.6 months.

\section{Discussion}

The efficacy assessment of chemotherapies for solid tumors is carried out in terms of tumor regression in accordance with the WHO criteria. There have been no definite international provisions for the efficacy assessment of chemotherapies for primary gastrointestinal lesions; efficacy assessment is done only for metastatic lesions in most patients. The possibility of assessing primary gastrointestinal lesions should be considered. Primary lesions were neglected in some reports on the anti-tumor efficacy of chemotherapies for gastric cancer from the United States and European countries. The possibility that primary lesions were not checked, despite their response being categorized as PD, cannot be ruled out. In any case, the assessment of primary gastric lesions is essential for the efficacy assessment of chemotherapies for gastric cancer. In Japan, assessment of primary lesions has already been carried out extensively. [9-12] Characteristically, even b-lesions - that is, lesions extending to more than half the circumference of the luminal organs that can not be measured and lesions with an unclear boundary — are examined care-

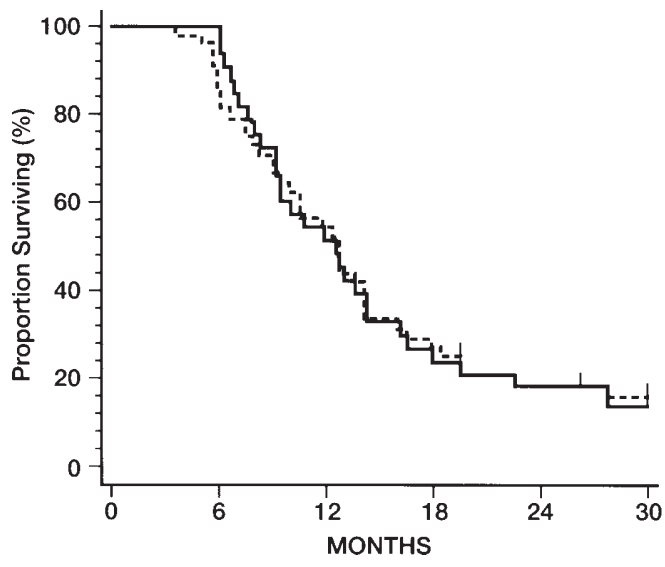

Fig. 4. Comparison of survival time of responders by Japanese criteria ${ }^{7}$ (Continuous line; $n=48$; MST, 12.6; 1-year survival, $54.2 \%$ ) and by WHO criteria ${ }^{4}$ (dashed line; $n=33$; MST, 12.6; 1-year survival, 51.5\%)

fully and comparatively, not only by upper gastrointestinal X-ray examination but also by endoscopy, before and after chemotherapy. Findings of tumor regression, defined as a definite $50 \%$ reduction, together with findings of a reduction in ulcerous area and a flattening of the surrounding bank, are determined to indicate PR. The objectivity of this assessment is maintained by extramural review. As for c-lesions, represented by linitis plastica, a unique assessment method is adopted, in which an enlargement of the affected area of more than $50 \%$ is determined to indicate PR. The PR rates in these assessments are lower than those for measurable lesions, which, however, indicates that a generally more rigorous determination was made. It can consequently be said that patients with b- or c-lesions who were assessed as showing PR exhibited very high anti-tumor efficacy with chemotherapies.

When we compare the survival of responders and non-responders, careful analyses and interpretations are necessary, taking statistical bias between them into account. Although some studies report that the antitumor efficacy of chemotherapies does not necessarily correlate with survival time, Ohtsu et al. [13] reported that, when they employed the Japanese criteria for efficacy evaluation, responders with primary gastric lesions showed significantly longer survival than nonresponders. Our study also showed that the MST of responders (12.6 months) was significantly longer than the MST of non-responders (7.6 months). Furthermore, the MSTs of responders classified by type of lesions were different (a-lesions, 14.2 months; b-lesions, 11.5 months; and c-lesions, 10.5 months) and the MSTs of non-responders were also different (a-lesions, 11.4 months; b-lesions, 7.4 months; and c-lesions, 7.2 months), suggesting that the lesions evaluated by the 
Japanese criteria may affect patients survival. Hazard ratios for each of the lesions, which quantify the difference in the above MSTs, showed similar values in responders (b-lesions, 1.79 and c-lesions, 2.19) and non-responders (b-lesions, 1.29 and c-lesions, 2.00 ), with these hazard ratios with a-lesions taken as 1.00 .

That is, it was suggested that anti-tumor efficacy assessment, in terms of evaluable but not measurable lesions and diffusely infiltrating lesions, showed a correlation with survival prognosis; This is comparable to the anti-tumor efficacy assessment of measurable lesions with tumor regression as an index of efficacy.

We compared the relationship between response to chemotherapy in patients with primary gastric lesions and survival prognosis, in terms of the WHO criteria and the Japanese criteria. According to the WHO criteria, only measurable lesions (a-lesions) can be evaluated, while both evaluable but not measurable lesions (b-lesions) and diffusely infiltrating lesions (c-lesions) are excluded from the evaluation. In this study, all 90 patients were evaluable by the Japanese criteria; however, only 49 of the 90 patients (54\%) were evaluable by the WHO criteria, because patients with b- or c-lesions, who have no measurable metastatic lesions, were excluded. In the 49 patients evaluable by the WHO criteria, the response rate was $67.3 \%$ (33/49), which was higher than that determined by the Japanese criteria $(53.3 \%$; 48/90). The MSTs of patients evaluable by the Japanese criteria and by the WHO criteria were the same, both for overall MST (9.4 months) and for the MST of responders (12.6 months).

The Japanese Criteria for Primary Gastric Cancer are generally used as the standard criteria for the evaluation of the efficacy of gastric cancer chemotherapy in Japan. Here, we have described the results of retrospective analyses, attempting to examine the relationship between anti-tumor effect, as the surrogate endpoint, and survival. We also compared the results of assessment by the Japanese criteria with the results of assessment by the WHO criteria.

Interestingly, although this was a retrospective pilot study, there was a positive relationship between antitumor effect for primary lesions and survival. We believe, therefore, that further, prospective, studies in patients who receive the same chemotherapy should be conducted to confirm our preliminary results. We also believe that efficacy evaluation by the Japanese criteria has an advantage over that by the WHO criteria, because patients who have only primary gastric lesions, which are not measurable by the WHO criteria, can be included for efficacy evaluation. In this study, 41 patients were excluded from efficacy evaluation by the WHO criteria, although they had inoperable gastric cancer, suggesting that certain specific lesions of gastric cancer are excluded from efficacy evaluation. Ideally, all patients with measurable/evaluable lesions should be included for efficacy evaluation. By the Japanese criteria, patients with lesions that are considered not measurable by the WHO criteria are included for efficacy evaluation. However, responders by the Japanese criteria showed the same MSTs as responders by the WHO criteria.

In conclusion, we suggest that the Japanese criteria are useful for evaluating the anti-tumor effect of chemotherapies and that prospective studies to reconfirm their usefulness are warranted in Japan, and in Western countries.

Acknowledgments. We are grateful to Masanori Shima and Takeshi Tahara for their assistance with the data analysis.

\section{References}

1. Carter SK. Study design principles for clinical evaluation for new drugs as developed by the chemotherapy program of the National Cancer Institute. In: Staquet M, editor. The design of clinical trials in cancer therapy. Brussels: Scientifiques Europeens, 1972:24289.

2. Oken MM, Creech RH, Tormey DC, Horton J, Davis TE, McFadden ET, et al. Toxicity and response criteria of the Eastern Cooperative Oncology Group. Am J Clin Oncol (CCT) 1982; 5:649-55.

3. Hayward JL, Carbone PP, Heuson JC, Kumaoka S, Segaloff A, Rubens RD. Assessment to response to therapy in advanced breast cancer. A project of the program on clinical oncology of the International Union against Cancer, Genova, Switzerland. Eur J Cancer 1977;13:89-94.

4. World Health Organization: WHO Handbook for reporting results of cancer treatment. WHO offset publication No.48: Genova, Switzerland, World Health Organization, 1979.

5. Japanese Research Society for Gastric Cancer. Japanese classification of gastric carcinoma (in Japanese). 11th ed. Tokyo: Kanehara; 1985.

6. Japanese Research Society for Gastric Cancer. Japanese classification of gastric carcinoma (in Japanese). 12th ed. Tokyo: Kanehara; 1993.

7. Japanese Research Society for Gastric Cancer. Japanese classification of gastric carcinoma. 1st English ed. Tokyo: Kanehara; 1995.

8. Cox DR. Regression models and life-tables. J R Stat Soc 1972; 34:187-202.

9. Kurihara M, Akiya T, Futatsuki K, Sasaki J, Yaosaka T, Betsuyaku T, et al. Evaluation of chemotherapeutic effect on advanced gastric cancer cases with primary focus by the criteria of chemotherapeutic effects on gastric cancer (in Japanese). Oncologia 1988;21:93-7.

10. Kurihara M, Izumi T, Ito $T$. Evaluation of the effect of chemotherapy in gastric cancer patients with an intact primary tumor according to the criteria of the Japanese Research Society for Gastric Cancer. J Jpn Soc Cancer Ther 1991:26;64454. 
11. Kurihara M, Matsukawa M. Evaluation of efficacy of chemotherapy / radiation therapy in the treatment of inoperative gastric cancer. In: Takahashi T, editor. Recent advances in management of digestive cancers. Berlin Heidelberg New York Tokyo: Springer-Verlag, 1993:132-7.

12. Koizumi W, Kurihara M, Hasegawa K, Chonan A, Kubo Y, Maekawa R, et al. Combination therapy with cisplatin, 5'- deoxy-5-fluorouridine (5'-DFUR) and mitomycin (MMC) in patients with inoperable, advanced gastric cancer: a randomized trial comparing two dosage regimens. Oncology Reports 1996;3:255-60.

13. Ohtsu A, Boku N, Yoshida S, Miyata Y, Shirao K, Shimada Y, et al. Response of the primary lesions in gastric cancer to chemotherapeutic trials. Int J Clin Oncol 1998;3:3-6. 